\title{
Granulocyte-colony stimulating factor producing mucinous cystic neoplasm with an associated invasive carcinoma of the pancreas
}

\author{
KAZUHIDE SHIMAMATSU ${ }^{1}$, YOSHIKI NAITO ${ }^{2}$, YUTARO MIHARA ${ }^{3}$, MASAMICHI NAKAYAMA $^{3}$, \\ MASAHIKO TANIGAWA ${ }^{3}$, YUSHI ABE $^{3}$, KEN NAKAMURA $^{3}$, TOSHIHIRO ARAKI $^{4}$, KENJI SAKATA $^{4}$, \\ KAZUNORI NOGUCHI $^{4}$, JUN AKIBA ${ }^{2}$, HIROHISA YANO ${ }^{3}$ and OSAMU NAKASHIMA ${ }^{5}$ \\ ${ }^{1}$ Department of Pathology, Omuta City Hospital, Omuta, Fukuoka 836-8567; ${ }^{2}$ Department of Diagnostic Pathology, \\ Kurume University Hospital; ${ }^{3}$ Department of Pathology, Kurume University School of Medicine, \\ Kurume, Fukuoka 830-0011; ${ }^{4}$ Department of Medicine, Omuta City Hospital, Omuta, Fukuoka 836-8567; \\ ${ }^{5}$ Department of Clinical Laboratory, Kurume University Hospital, Kurume, Fukuoka 830-0011, Japan
}

Received July 1, 2017; Accepted October 26, 2017

DOI: $10.3892 / \mathrm{ol} .2017 .7572$

\begin{abstract}
The present case study documents an autopsy case of granulocyte-colony stimulating factor (G-CSF)-producing mucinous cystic neoplasm (MCN), with an associated invasive carcinoma of the pancreas. A 65-year-old woman presented to Omuta City Hospital (Omuta Japan) with a primary complaint of abdominal pain. Multiple liver nodules and a pancreatic cyst were detected upon abdominal computed tomography. Initially, liver abscess was suspected as the patient exhibited leukocytosis and elevated C-reactive protein level. However, the serum concentration of G-CSF was $98.8 \mathrm{pg} / \mathrm{ml}$ (normal, $<39.0 \mathrm{pg} / \mathrm{ml}$ ). At 6 weeks after admission, the patient succumbed to liver failure. At autopsy, a cystic lesion was identified in the pancreatic tail that contained bloody necrotic fluid. Microscopically, the cystic lesion was composed of columnar and mucin-producing epithelium associated with ovarian-type subepithelial stroma. The stroma exhibited positive immunostaining for vimentin, estrogen receptor and progesterone receptor. Calcification on the cystic wall was observed. The tumor invaded the pancreatic parenchyma and metastasized to the liver and lungs. The lesion was diagnosed as invasive adenocarcinoma arising in MCN. By contrast, liver nodules predominantly consisted of pleomorphic cancer cells with small foci of adenocarcinoma. Pancreatic and hepatic cancer cells were confirmed to be positive for G-CSF staining. The present case report indicates that G-CSF-producing
\end{abstract}

Correspondence to: Dr Kazuhide Shimamatsu, Department of Pathology, Omuta City Hospital, 2-19-1 Takarazakamachi, Omuta, Fukuoka 836-8567, Japan

E-mail: kshima1693@ghp.omuta.fukuoka.jp

Abbreviations: G-CSF, granulocyte colony-stimulating factor; MCN, mucinous cystic neoplasm; PgR, progesterone receptor; ER, estrogen receptor

Key words: pancreas, mucinous cystic neoplasm, anaplastic change, granulocyte-colony stimulating factor, autopsy case
MCNs may be associated with an aggressive clinical course, particularly when anaplastic changes are observed.

\section{Introduction}

Granulocyte-colony stimulating factor (G-CSF) is a glycoprotein associated with the proliferation and maturation of neutrophils $(1,2)$. Since the first case of G-CSF-producing lung carcinoma reported by Asano et al in 1977 (3), similar carcinomas have been reported in various organs $(4,5)$. However, G-CSF-producing carcinomas of the pancreas are relatively rare (6-10). Mucinous cystic neoplasms (MCN) are defined as cyst-forming epithelial neoplasms that arise in the pancreas (11). MCNs have a female predominance, and they arise in the body and tail of the pancreas, without communication with the pancreatic duct system (12). The most specific pathological feature of MCNs is ovarian-type subepithelial stroma in the cyst wall (12). According to previous reports, the ovarian-like stroma of MCNs exhibits immunohistochemically positive progesterone receptor (PgR) and estrogen receptor (ER) (13). The size of MCNs ranges between 2 and $35 \mathrm{~cm}$ in diameter, and patients with MCNs with an associated invasive carcinoma are 5-10 years older compared with those with non-invasive MCNs (11). These findings indicate that the progression from non-invasive $\mathrm{MCN}$ to invasive carcinoma occurs over a period of years. The histology of G-CSF-producing neoplasms of the pancreas, including unconventional tumors such as adenosquamous carcinoma or anaplastic carcinoma, has been previously described $(6,7,9,10,14,15)$. However, to the best of our knowledge, there are no reports concerning MCNs, and this is the first study to identify positive G-CSF immunostaining in a patient with MCN with an associated invasive carcinoma.

\section{Case report}

A 65-year-old woman presenting with abdominal pain, constipation and lingering fever was referred to Department of Medicine, Omuta City Hospital (Omuta, Japan) for further examination in April 2015. Abdominal computed tomography indicated multiple hepatic nodules, a pancreatic cyst and 

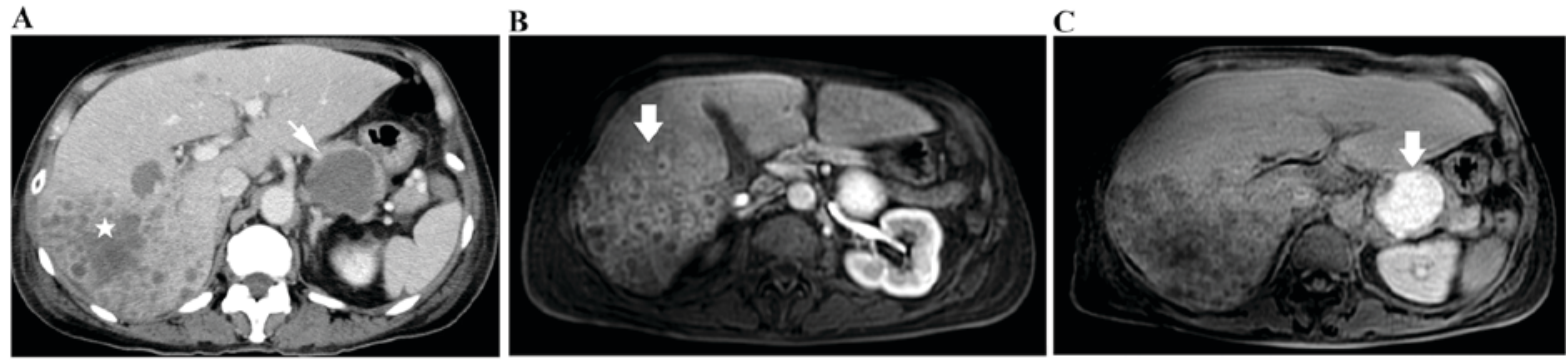

Figure 1. Abdominal computed tomography and magnetic resonance imaging findings. (A) Multiple liver nodules (arrowhead) and a single cystic lesion in the pancreatic tail (arrow). (B) Numerous ring-enhancing lesions in the liver (arrow). (C) A pancreatic tail cyst with high intensity (arrow) on a fat suppression T1-weighted image.

ascites (Fig. 1A). The abdominal magnetic resonance imaging indicated multiple ring-enhanced lesions (Fig. 1B), and a pancreatic tail cyst exhibited high intensity on a fat suppression T1-weighted image (Fig. 1C). Laboratory tests revealed marked leukocytosis [white blood cell count, 39,640/ $\mu$ 1 (normal, 3,500-9,100/ $\mu 1$ ); neutrophils, 94.1\% (normal, 32-79\%); monocytes, $3.2 \%$ (normal, $0-8 \%$ ); lymphocytes, $2.3 \%$ (normal, 18-59\%); eosinophils, $0.3 \%$ (normal, 0-6\%); basophils, $0.1 \%$ (normal, 0-2\%); and no blasts] and elevated serum C-reactive protein level (20.4 mg/dl; normal, $<0.3 \mathrm{mg} / \mathrm{dl})$. Serum tumor markers, including carbohydrate antigen 19-9 (10.5 U/ml; normal 0-37 U/ml), carcinoembryonic antigen $(3.2 \mathrm{ng} / \mathrm{ml}$; normal $0-5 \mathrm{ng} / \mathrm{ml}$ ) and $\alpha$-fetoprotein $(1.8 \mathrm{ng} / \mathrm{ml} ; 0-10 \mathrm{ng} / \mathrm{ml})$, were within normal limits. Ascites cytology was negative for malignancy. Bone marrow aspiration revealed hypercellular marrow with excessive myeloid cells. Considering the clinical symptoms, radiological studies and laboratory results, liver abscess was suspected as an infective focus. However, neither arterial infusion therapy of imipenem/cilastatin $(1 \mathrm{~g} /$ day for 4 days) nor administration of tazobactam/piperacillin (13.5 g/day for 10 days), metronidazole $(1,500 \mathrm{mg} /$ day for 10 days) and amphotericin B (2 g/day for 4 days) elicited any response in the patient. Upon further examination, the cystic lesion in the pancreas was suspected to be a malignant neoplasm, and tumor-associated leukocytosis was considered. The serum concentration of G-CSF was elevated $(98.8 \mathrm{pg} / \mathrm{ml}$; normal $<39.0 \mathrm{pg} / \mathrm{ml}$ ). The physical status of the patient deteriorated rapidly, with multiple liver nodules and aggravation of jaundice and ascites. At 6 weeks after admission, the patient succumbed to liver failure. Written informed consent was obtained from the patient's family following mortality.

At autopsy, a cystic lesion (size, $4 \times 3 \mathrm{~cm}$ ) was noted in the pancreatic tail. This lesion had an elastic hard wall and contained bloody necrotic fluid (Fig. 2A). Multiple liver nodules, located predominantly in the right lobe, were observed (Fig. 2B). Sections (3- $\mu$ m thick) were fixed with $10 \%$ formalin and paraffin-embedded at room temperature for 2 days. The sections were stained with hematoxylin for $5 \mathrm{~min}$ and with eosin for $3 \mathrm{~min}$ at room temperature. Histologically, the pancreatic tumor was a cystic lesion that had ovarian-type subepithelial stroma with focal calcification and was lined by columnar mucinous epithelium with high-grade dysplasia (Fig. 3A-C). Adenocarcinoma, which extended into pancreatic acinus and invaded splenic vein, exhibited irregular glandular structures and poorly cohesive cell clusters (Fig. 4A and B). The parenchyma was infiltrated by considerable neutrophils (Fig. 4B).

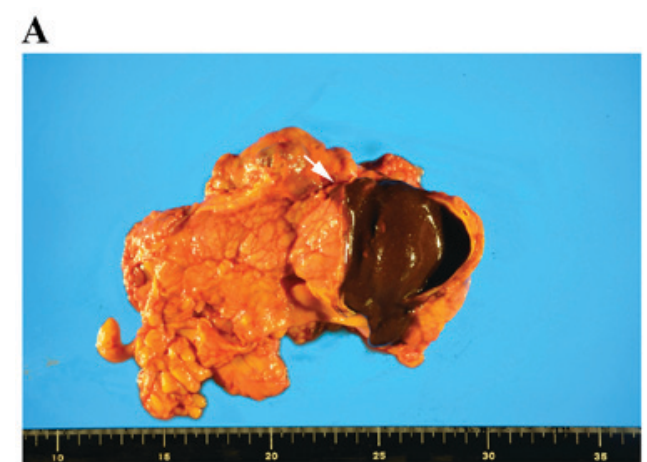

B

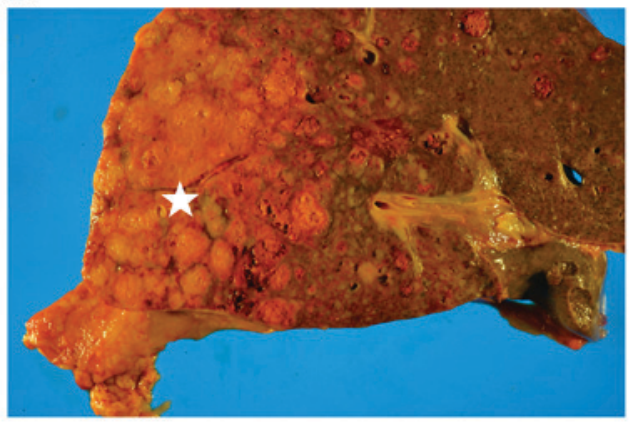

Figure 2. Macroscopic findings of the pancreas and liver. (A) A 4x3 cm sized cystic lesion with bloody necrotic fluid (arrow). (B) Multicentric metastases in the liver (star).

Sections (3- $\mu \mathrm{m}$ thick) were fixed with $10 \%$ formalin and paraffin-embedded at room temperature for 2 days. Onboard heat-induced antigen retrieval was performed on a fully automated BOND-III system (Leica Microsystems Ltd., Milton Keynes, UK) with a BOND Epitope Retrieval Solution 2 (cat. no. AR9640; Leica Biosystems, Inc., Buffalo Grove, IL, USA) for $20 \mathrm{~min}$ at $99^{\circ} \mathrm{C}$, and sections were incubated with the following primary antibodies for $15 \mathrm{~min}$ at room temperature: Mucin (MUC)1 (cat. no. NCL-MUC-1; dilution, 1:300; clone Ma695; Novocastra; Leica Biosystems, Inc.), MUC2 (cat. no. NCL-MUC-2; dilution, 1:200; clone Ccp58; Novocastra; Leica Biosystems, Inc.), MUC5AC (cat. no. NCL-MUC-5AC; dilution, 1:200; clone CLH2; Novocastra; Leica Biosystems, Inc.), cytokeratin AE1/AE3 (cat. no. N3515; dilution, 1:300; clone AE1/AE3; Dako; Agilent Technologies, Inc., Santa Clara, CA, USA), Vimentin (cat. no. M0725; dilution 1:9; clone V9; Dako; Agilent Technologies, Inc.) and G-CSF (cat. no. GF05; dilution 1:2,000; clone 5.24; Calbiochem; EMD Millipore, 

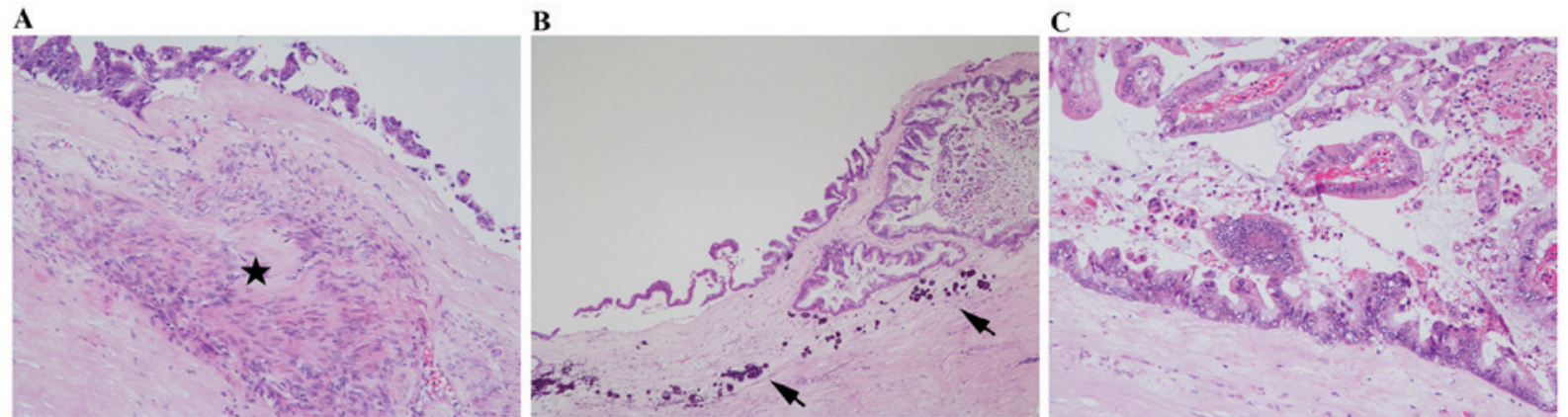

Figure 3. Microscopic findings of mucinous cystic neoplasm with an associated invasive carcinoma. (A) Ovarian-type subepithelial stroma (star) (H\&E staining; magnification, $\mathrm{x} 40$ ) and (B) focal calcification in the cyst wall (H\&E staining; magnification, $\mathrm{x} 40$ ). (C) Columnar mucinous epithelium with high-grade dysplasia (H\&E staining; magnification, x100).

A

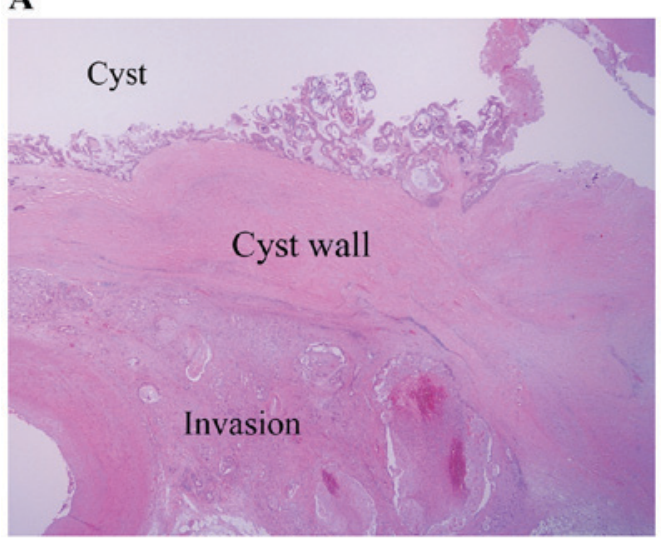

B

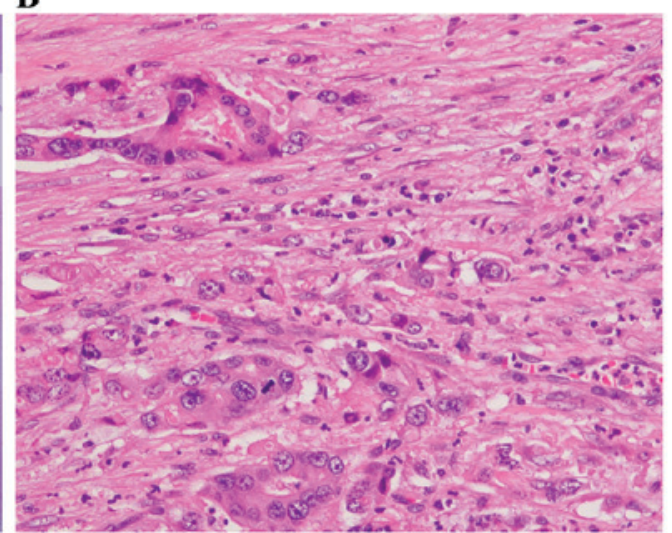

Figure 4. Parenchymal invasion of the pancreatic tumor. (A) Tumor cells invaded into pancreas (H\&E staining; magnification, x10). (B) Invasive adenocarcinoma surrounded by considerable neutrophils was observed in the pancreas (H\&E staining; magnification, x200).

A

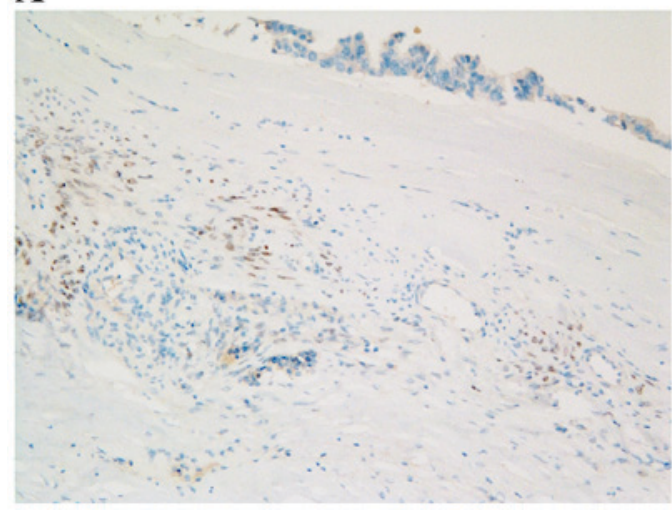

B

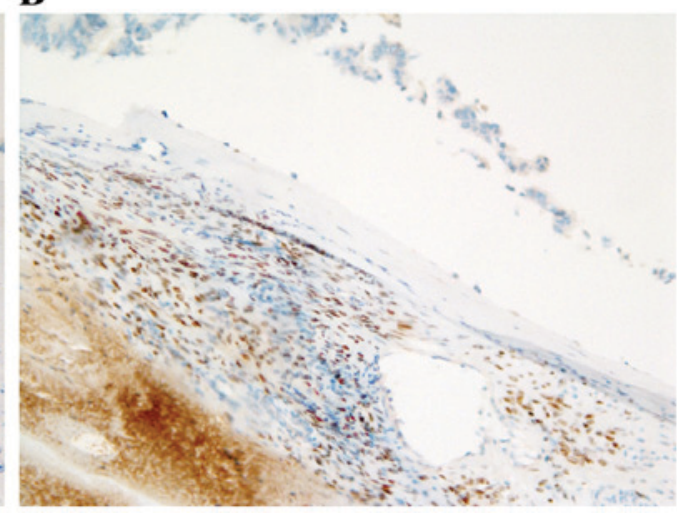

Figure 5. Immunohistochemical findings of ovarian-type subepithelial stroma. Expression of (A) estrogen receptor and (B) progesterone receptor was observed in ovarian-type subepithelial stroma cells (magnification, x100).

Billerica, MA, USA). Peroxide Blocking Reagent (3-4\%) (cat. no. DS9800; Leica Biosystems, Inc.) was applied for 5 min at room temperature, according to the manufacturer's protocols. The automated system used a Bond Polymer Refine Detection kit (cat. no. DS9800; Leica Biosystems, Inc.; containing an immunoglobulin $\mathrm{G}$ linker, a horseradish peroxidase-linked polymer and 3,3'-diaminobenzidine (DAB) to detect the bound primary antibodies. Incubation with the secondary antibody was performed for $30 \mathrm{~min}$ at room temperature.
ER (cat. no. 107925; ready to use; clone SP1; Ventana Medical Systems, Inc., Tucson, AZ, USA) and PgR (cat. no. 102333; ready to use; clone 1E2; Ventana Medical Systems, Inc.) staining for $32 \mathrm{~min}$ at room temperature was performed using BenchMark ULTRA (Ventana Medical Systems, Inc.). The automated system used the streptavidin-biotin complex method with DAB as a chromogen (cat. no. 109431; Ventana iVIEW DAB Detection kit; Ventana Medical Systems, Inc.). An Olympus BX51 optical 
A

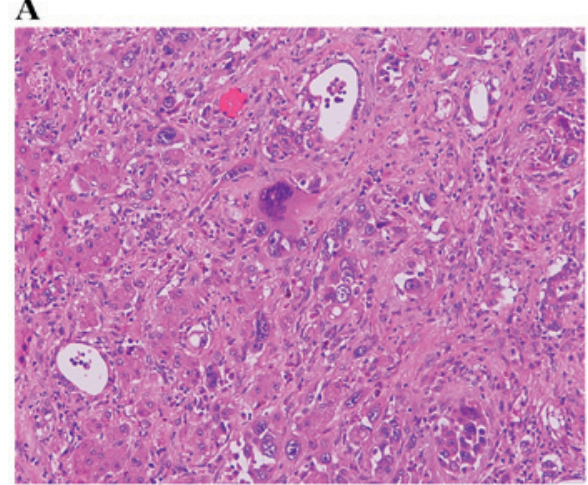

C

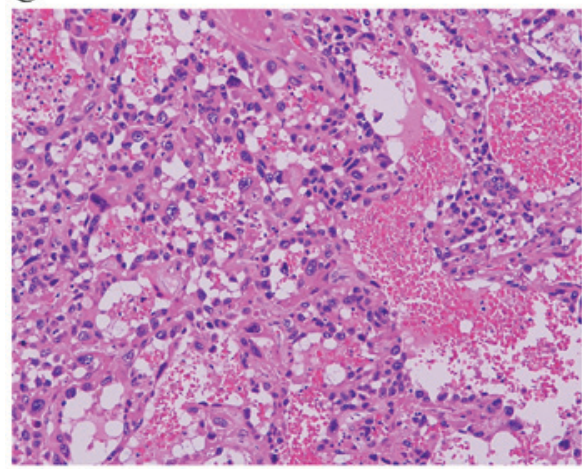

B

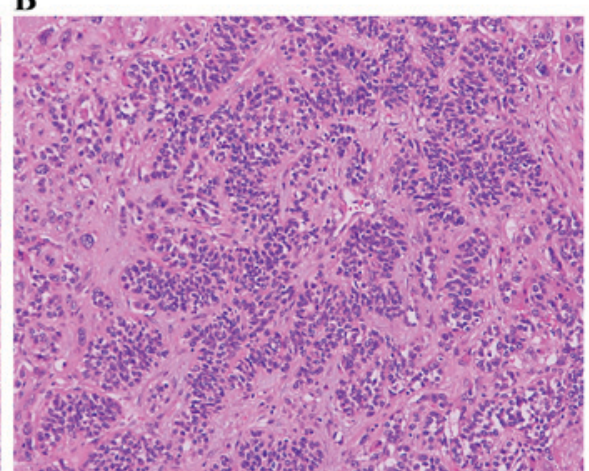

D

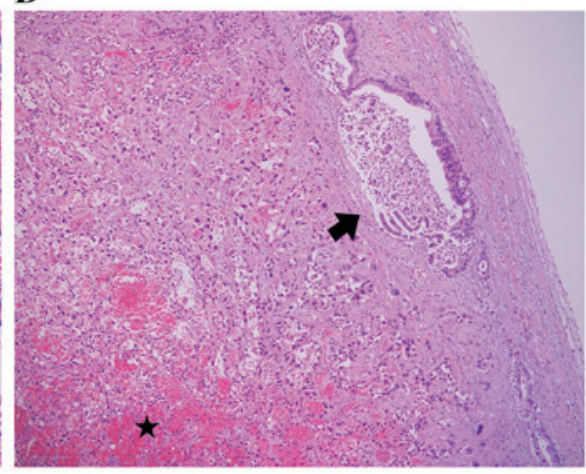

Figure 6. Histological and immunohistochemical findings of liver metastasis. (A) Anaplastic cells (magnification, x100), (B) trabecular structure (magnification, $\mathrm{x} 100$ ) and (C) angiosarcoma-like appearance (magnification, x100). (D) Adenocarcinoma foci (arrow) and necrotic background (star) (magnification, $\mathrm{x} 40$ ).

A

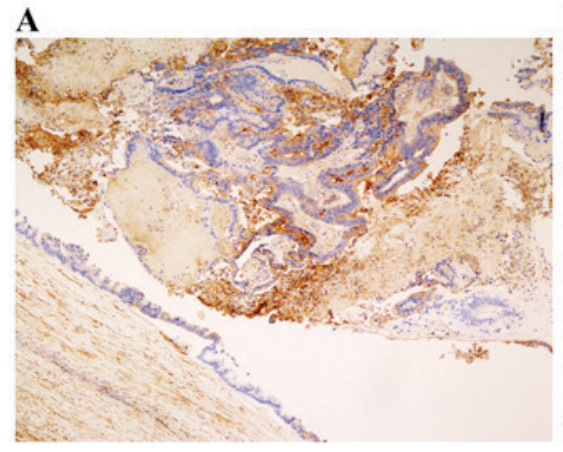

B

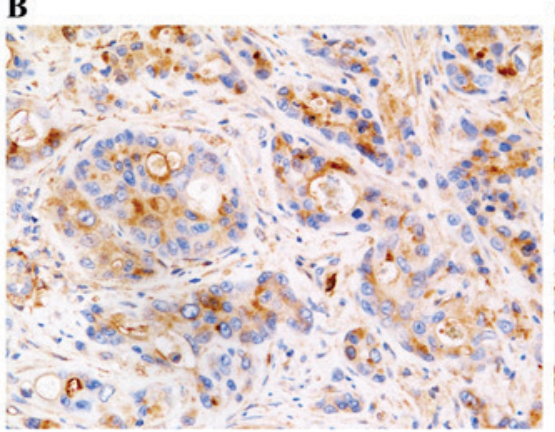

C

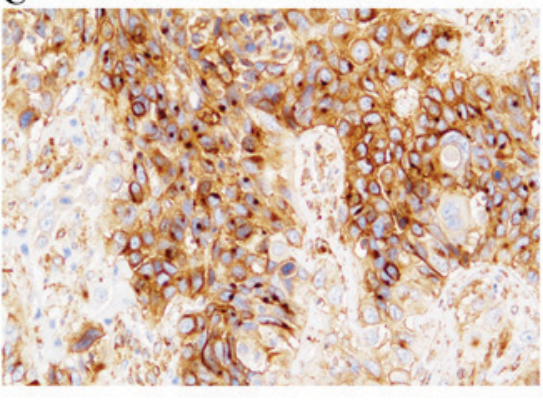

Figure 7. Positive immunostaining for anti-G-CSF receptor antibody. G-CSF staining was positive for (A) pancreatic intraepithelial neoplasia (H\&E staining; magnification, x40), (B) invaded adenocarcinoma (H\&E staining; magnification, x200) and (C) liver metastatic cancer cells (H\&E staining; magnification, $\mathrm{x} 200)$. G-CSF, granulocyte-colony stimulating factor.

microscope was used to view all slides under $\mathrm{x} 12.5-400$ magnification.

The tumor cells exhibited positive staining for MUC5AC, and negative staining for MUC1 and MUC2. The ovarian-type subepithelial stroma exhibited positive staining for vimentin, ER and PgR (Fig. 5A and B). On the basis of these findings, the tumor was diagnosed as a MCN with an associated invasive carcinoma of the pancreas.

Lymph nodes of the hepatic portal region were swollen and white/off-white masses (size, 2-3 mm) were identified scattered in the lungs. Microscopically, all of the lymph nodes exhibited moderately to poorly differentiated adenocarcinoma. By contrast, the hepatic nodules consisted of pleomorphic cells, represented by anaplastic giant cells, a trabecular growth pattern and an angiosarcoma-like appearance, along with small adenocarcinoma foci beneath the liver capsule and a massive necrotic background (Fig. 6A-D). The immunological profile of the adenocarcinoma was identical to that of the pancreatic MCN. The angiosarcomatous area was positive for cytokeratin (AE1/AE3) and vimentin. The pancreatic and hepatic cancer cells were confirmed to be positive for immunostaining of G-CSF (Fig. 7A-C).

\section{Discussion}

Following the purification of human G-CSF from tumor cell lines (16) and its utilization for immunoperoxidase staining of paraffin-embedded sections (17), several G-CSF-secreting pancreatic carcinomas have been confirmed using immunohistochemistry (6). The diagnostic criteria for G-CSF-producing 
tumors are as follows: i) Extreme leukocytosis, ii) elevated G-CSF activity, iii) a decreased white blood cell count following tumor resection, or iv) proof of G-CSF production in the tumor (9). The detection of G-CSF by immunostaining is difficult as the G-CSF protein is generally retained in the cytoplasm for a short time and the antigenicity is inconstant (18). Although, the intensity of G-CSF immunostaining varied by location in the case discussed in the present study, positive staining was easily recognized in intraepithelial neoplastic cells, invaded adenocarcinoma and liver metastatic cells. Thus, it was concluded that present case is a rare, G-CSF-producing tumor.

Carcinomas that produce G-CSF are known to be highly malignant tumors $(8,10)$. G-CSF functions as not only a hematopoietic growth factor but also as an autocrine growth factor associated with the proliferation of tumor cells $(19,20)$. G-CSF released by tumor cells may bind to G-CSF receptors in the tumor cells, triggering proliferation, invasion, migration via an autocrine mechanism, and the transformation of the epithelial elements into a more immature or high-grade phenotype $(16,19)$. Regardless of the therapeutic efforts, the prognosis of patients G-CSF-producing pancreatic tumors is extremely poor, which is supported by the fact that all referred cases, regardless of surgical treatment or chemotherapy, succumbed to disease within 8 months of the initial consultation (12).

Histologically, anaplastic carcinoma $(7,10,14,15)$, poorly differentiated adenocarcinoma (8), and adenosquamous carcinoma $(6,9)$ are the dominant subtypes of G-CSF-producing pancreatic tumor. To the best of our knowledge, an incidence of MCN with associated invasive carcinoma of the pancreas has not been reported in the English or Japanese literature. MCNs typically present as a single, spherical mass with a smooth surface and a fibrous pseudocapsule of variable thickness with occasional calcification (21). Judging from the scattered calcification and scarce ovarian-type subepithelial stroma, the case discussed in the present study is a long-term MCN, from which malignant transformation of lining columnar cells and invasive carcinoma may develop.

Metastatic cancer cells in the liver, the majority of which were positive for G-CSF immunostaining, exhibited pleomorphism. It is unclear why anaplastic change was observed only in the liver. Liver biopsy was not performed in the initial period of admission due to the presence of ascites and a risk of hemorrhage. Although metastatic liver cancer was indicated by radiological findings, arterial infusion of antibiotics was conducted on the presumptive diagnosis of liver abscess. It is arguable whether the antibiotic therapy contributed to the alternation of cellular morphology. For example, the procedure propagated the sinusoidal spread of carcinoma cells and formed the angiosarcomatous vasoformative architecture. However, the tumor cells were positive for cytokeratin and vimentin. As for anticancer drugs, the majority of sarcomatoid changes in hepatocellular carcinoma are associated with repeated chemotherapy or transarterial chemoembolization (22). However, to the best of our knowledge, the same transformation induced by antibiotics has not been reported. It can therefore be concluded that anaplastic change was associated with nature of the tumor rather than the procedure.

Unlike those with non-invasive MCNs, patients with invasive MCNs are considered to have a poor prognosis (23), and also in the present case, the invasive cancer led to mortality following an aggressive clinical course. A case report alone may not be sufficient to draw a firm conclusion. However, G-CSF-producing MCN with an associated invasive carcinoma of the pancreas may be at type of pancreatic cancer with a poor prognosis, particularly when poorly differentiated carcinoma or sarcomatous changes are observed.

\section{References}

1. Lieschke GJ and Burgess AW: Granulocyte colony-stimulating factor and granulocyte-macrophage colony-stimulating factor (1). N Engl J Med 327: 28-35, 1992.

2. Lieschke GJ and Burgess AW: Granulocyte colony-stimulating factor and granulocyte-macrophage colony-stimulating factor (2). N Engl J Med 327: 99-106, 1992.

3. Asano S, Urabe A, Okabe T, Sato N and Kondo Y: Demonstration of granulopoietic factor(s) in the plasma of nude mice transplanted with a human lung cancer and in the tumor tissue. Blood 49: 845-852, 1977.

4. Toyoda M, Chikamatsu K, Sakakura K, Fukuda Y, Takahashi K, Miyashita M, Shimamura K and Furuya N: A case of squamous cell carcinoma of the head and neck producing granulocyte-colony stimulating factor with marked leukocytosis. Auris Nasus Larynx 34: 267-271, 2007.

5. Nakayama K, Takahashi T, Tsuyuoka R, Ueda Y, Suzuki A, Okuno Y, Ihara Y, Seko S, Okada T, Kumagai N, et al: Identification of colony-stimulating factor activity in patients with malignant tumors associated with excessive leukocytosis. Jpn J Clin Oncol 21: 395-399, 1991.

6. Ohtsubo K, Mouri H, Sakai J, Akasofu M, Yamaguchi Y, Watanabe H, Gabata T, Motoo Y, Okai T and Sawabu N: Pancreatic cancer associated with granulocyte-colony stimulating factor production confirmed by immunohistochemistry. J Clin Gastroenterol 27: 357-360, 1998.

7. Gotohda N, Nakagohri T, Saito N, Ono M, Sugito M, Ito M, Inoue K, Oda T, Takahashi S and Kinoshita T: A case of anaplastic ductal carcinoma of the pancreas with production of granulocyte-colony stimulating factor. Hepatogastroenterology 53: 957-959, 2006.

8. Takami K, Miura K, Takeuchi H, Egawa S, Moriya T, Nakamura Y, Tanabe A, Sugita J, Karasawa H, Unno M and Sasaki I: Granulocyte-colony stimulating factor-producing pancreatic cancer: Report of a case. Surg Today 38: 453-457, 2008.

9. Joshita S, Nakazawa K, Sugiyama Y, Kamijo A, Matsubayashi K, Miyabayashi H, Furuta K, Kitano K and Kawa S: Granulocyte-colony stimulating factor-producing pancreatic adenosquamous carcinoma showing aggressive clinical course. Intern Med 48: 687-691, 2009.

10. Kitade H, Yanagida H, Yamada M, Satoi S, Yoshioka K, Shikata $\mathrm{N}$ and Kon $\mathrm{M}$ : Granulocyte-colony stimulating factor producing anaplastic carcinoma of the pancreas treated by distal pancreatectomy and chemotherapy: Report of a case. Surg Case Rep 1: 46, 2015.

11. Kosmahl M, Pauser U, Peters K, Sipos B, Luttges J, Kremer B and Klöppel G: Cystic neoplasms of the pancreas and tumor-like lesions with cystic features: A review of 418 cases and a classification proposal. Virchows Arch 445: 168-178, 2004.

12. Tanaka M, Chari S, Adsay V, Fernandez-del Castillo C, Falconi M, Shimizu M, Yamaguchi K, Yamao K and Matsuno S; International Association of Pancreatology: International consensus guidelines for management of intraductal papillary mucinous neoplasms and mucinous cystic neoplasms of the pancreas. Pancreatology 6: 17-32, 2006.

13. Fukushima N and Fukayama M: Mucinous cystic neoplasms of the pancreas: Pathology and molecular genetics. J Hepatobiliary Pancreat Surg 14: 238-242, 2007.

14. Murata T, Terasaki M, Sakaguchi K, Okubo M, Fukami Y, Nishimae K, Kitayama Y and Hoshi S: A case of anaplastic carcinoma of the pancreas producing granulocyte-colony stimulating factor. Clin J Gastroenterol 2: 109-114, 2009.

15. Nakajima A, Takahashi H, Inamori M, Abe Y, Kobayashi N, Kubota K and Yamanaka S: Anaplastic carcinoma of the pancreas producing granulocyte-colony stimulating factor: A case report. J Med Case Rep 2: 391, 2008.

16. Nomura H, Imazeki I, Oheda M, Kubota N, Tamura M, Ono M, Ueyama Y and Asano S: Purification and characterization of human granulocyte colony-stimulating factor (G-CSF). EMBO J 5: 871-876, 1986 . 
17. Shimamura K, Fujimoto J, Hata J, Akatsuka A, Ueyama Y, Watanabe T and Tamaoki N: Establishment of specific monoclonal antibodies against recombinant human granulocyte colony-stimulating factor (hG-CSF) and their application for immunoperoxidase staining of paraffin-embedded sections. J Histochem Cytochem 38: 283-286, 1990.

18. Takenaka M, Akiba J, Kawaguchi T, Niizeki T, Arinaga-Hino T, Sata M, Nakashima O, Yano $\mathrm{H}$ and Kage M: Intrahepatic cholangiocarcinoma with sarcomatous change producing granulocyte-colony stimulating factor. Pathol Int 63: 233-235, 2013.

19. Mueller MM, Herold-Mende CC, Riede D, Lange M, Steiner HH and Fusenig NE: Autocrine growth regulation by granulocyte colony-stimulating factor and granulocyte macrophage colony-stimulating factor in human gliomas with tumor progression. Am J Pathol 155: 1557-1567, 1999

20. Tachibana M, Miyakawa A, Tazaki H, Nakamura K, Kubo A Hata J, Nishi T and Amano Y: Autocrine growth of transitional cell carcinoma of the bladder induced by granulocyte-colony stimulating factor. Cancer Res 55: 3438-3443, 1995.
21. Zamboni G,Fukushima N,Hruban RH and Kloppel G: Mucinous cystic neoplasm of the pancreas. In: World health organization classification of tumors. WHO classification of tumor of the digestive system. Bosman FT, Carneiro F, Hruban RH and Theise ND (eds). IARC Press, Lyon, pp300-303, 2010.

22. Kojiro M, Sugihara S, Kakizoe S, Nakashima O and Kiyomatsu K: Hepatocellular carcinoma with sarcomatous change: A special reference to the relationship with anticancer therapy. Cancer Chemother Pharmacol 23 (Suppl): S4-S8, 1989.

23. Wilentz RE, Albores-Saavedra J, Zahurak M, Talamini MA, Yeo CJ, Cameron JL and Hruban RH: Pathologic examination accurately predicts prognosis in mucinous cystic neoplasms of the pancreas. Am J Surg Pathol 23: 1320-1327, 1999. 